\title{
Lossless Wide-Band Reflective Star Coupler Using Erbium-Doped Fiber Amplifiers
}

\author{
Jen-Yu Zeng, Wann-Yih Guo, and Yung-Kuang Chen
}

\begin{abstract}
A wide-band 64-port amplified reflective star coupler (ARSC) using eight fiber amplifiers and only four pump laser diodes is demonstrated. The loss of this ARSC can be fully compensated from $1530 \mathrm{~nm}$ to $1565 \mathrm{~nm}$ due to its double-pass amplification process. The power margin increment of $21.7 \mathrm{~dB}$ with the power penalty of $1.3 \mathrm{~dB}$ and the cross-channel power penalty of $2 \mathrm{~dB}$ are achieved in a $1.7 \mathrm{~Gb} / \mathrm{s}$ system experiment. This ARSC technology makes the star coupler cost-effective to be used in many larger wavelength division multiple access networks.
\end{abstract}

\section{INTRODUCTION}

$\mathbf{O}^{2}$ PTICAL $N \times N$ star networks have the potential to provide very large throughput, and they are expected to find widespread applications in upgrading existing networks and photonic networks [1]-[3]. However, the inherent $1 / N$ splitting and excess losses may limit the ultimate size and throughput of the star network. Some researches, with incorporation of erbium-doped fiber amplifiers (EDFA's) for accommodating more users, $N$, had been shown in transmissive star couplers [5]-[8]. On the other hand, the number of fibers and components required to implement a star network can be reduced to half by using an $N$-port reflective star coupler instead of a transmissive $N \times N$ star coupler [4]. Thus, besides cost-effectiveness, the reflective star coupler incorporating bidirectional EDFA's, whose double-pass amplification we need is expected to have higher signal gain, higher pump power use efficiency, and finally larger network size when compared with the transmissive one. It has applications in many wavelength division multiple access (WDMA) networks.

In the letter, we present the first, to our knowledge, amplified reflective star coupler (ARSC). The loss of the 64-port ARSC can be easily and fully compensated in a wide spectral range using eight EDFA's pumped with only four 980-nm laser diodes. The characteristics and features of the ARSC are investigated and discussed.

\section{AMPLIFIED REFLECTIVE STAR COUPLER}

The schematic diagram of the 64-port ARSC is shown in Fig. 1. It consists of eight, $8 \times 1$ tree couplers served as 64 input/output $(I / O)$ ports, eight EDFA's, an $8 \times 1$ tree coupler, and a fiber reflector mirror. Not including the losses of unpumped EDF and WDM coupler, the total passive loss for every $I / O$ port of this scheme has an average value of

Manuscript received June 13, 1994; revised September 6, 1994.

The authors are with Telecommunication Laboratories, Ministry of Transportation and Communications, P. O. Box 6-30, Yang-Mei 326, Taiwan, Republic of China.

They are also affiliated with the Institute of Electro-Optical Engineering, National Chiao-Tung University, Taiwan.

IEEE Log Number 9406596.

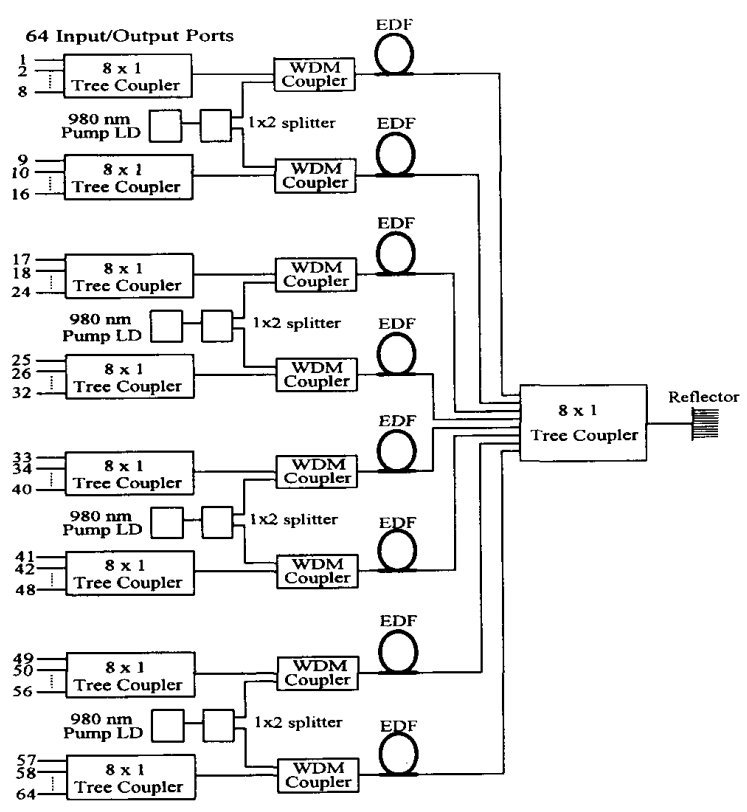

Fig. 1. The schematic diagram of the 64-port amplified reflective star coupler (ARSC).

$\sim 37.8 \mathrm{~dB}$ that is composed of $\sim 18.8 \mathrm{~dB}$ for one-pass travel and $\sim 0.2 \mathrm{~dB}$ for the reflector. It is this loss we feel like fully compensating. Each homemade EDF we used has a core size of $4.2 \mu \mathrm{m}$ and a numerical aperture (NA) of 0.23 . Every two EDFA's is pumped by a single pump laser diode, and there are totally four $980-\mathrm{nm}$ pump laser diodes used in this scheme. Each pump laser with $\sim 60 \mathrm{~mW}$ output power is coupled into a $1 \times 2980-\mathrm{nm}$ splitter, then only $\sim 30 \mathrm{~mW}$ pump power is launched into the $11.5 \mathrm{~m}$ EDF through a $0.98 / 1.55 \mu \mathrm{m}$ WDM coupler. Using an $8 \times 1$ tree coupler, we combine the eight branches of preceding eight $8 \times 1$ tree couplers and use only one reflector to turn the signal and residual pump power back.

In the experiments, because of the lack of pump laser diodes, the loss and noise figure (NF) characteristics of this 64-port ARSC were measured at the situations that the upper one, two, or four EDFA's were activated, respectively. On condition that four EDFA's are activated, a pigtailed $1552-\mathrm{nm}$ DFB laser transmitter is modulated by a $1.7-\mathrm{Gb} / \mathrm{s}\left[2^{31}-1\right]$ NRZ pseudorandom data stream, and the receiver used is composed of a 0.4-nm optical bandpass filter and an avalanche photodiode that has $-35.8 \mathrm{dBm}$ sensitivity for $10^{-9}$ bit-errorrate (BER) at $1.7 \mathrm{~Gb} / \mathrm{s}$. 


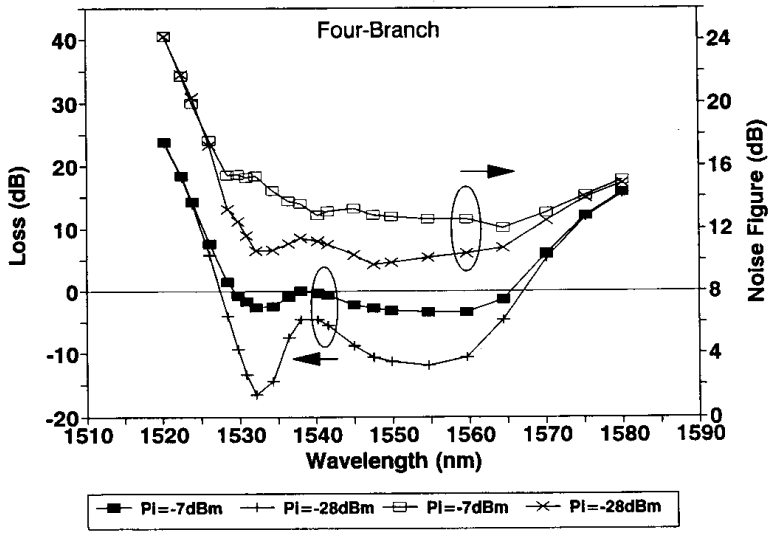

Fig. 2. The spectral loss and noise figure of this ARSC for small and large input signals.

\section{EXPERIMENTAL RESULTS}

The characteristics measured below are all at the situation where four EDFA's are activated (i.e., four-branch case) unless other conditions are mentioned explicitly. The signal loss is measured at the $I / O$ port; nevertheless, the NF measured is defined at the point just prior to the EDF, and thus it includes only the EDF, the right-hand side $8 \times 1$ tree coupler, and the reflector. Fig. 2 shows the spectral loss and NF characteristics of this ARSC for a single input signal at channel \#1 with small signal $(-28 \mathrm{dBm})$ and large signal $(-7 \mathrm{dBm})$ input powers (i.e., Pi). The small signal gain is $\sim 17 \mathrm{~dB}$ at $1532 \mathrm{~nm}$, which is slightly higher than that in the $1550 \mathrm{~nm}$ range. For large input signal, the variations of system net gain are less than 3.5 $\mathrm{dB}$ in the spectral range from $1530 \mathrm{~nm}$ to $1565 \mathrm{~nm}$. Note that the system double-pass loss can be fully compensated from $1530 \mathrm{~nm}$ to $1565 \mathrm{~nm}$ when the input signal power is less than $-7 \mathrm{dBm}$. The NF ranges from $9.7 \mathrm{~dB}$ to $11.5 \mathrm{~dB}$ and from $12 \mathrm{~dB}$ to $15.3 \mathrm{~dB}$ for small and large input signal powers, respectively, in the same spectral range, which is worse than that of the transmissive cases [8] by reason of the excess ASE noise generated in the double-pass amplification process of the EDFA.

Fig. 3 represents the spectral loss and NF characteristics of this ARSC with constant input power of $-18 \mathrm{dBm}$ for one-branch and four-branch cases. For four-branch cases, the system double-pass net gain is $\sim 12 \mathrm{~dB}$ at $1532 \mathrm{~nm}$ and $\sim 10$ $\mathrm{dB}$ at $1550 \mathrm{~nm}$; NF is $12.4 \mathrm{~dB}$ and $10.6 \mathrm{~dB}$ at $1532 \mathrm{~nm}$ and $1550 \mathrm{~nm}$, respectively. It means the internal optical gain is $\sim 50$ $\mathrm{dB}$ at $1532 \mathrm{~nm}$ and $\sim 48 \mathrm{~dB}$ at $1550 \mathrm{~nm}$; therefore, the ratio of internal gain to pump power of each EDFA is $\sim 48 \mathrm{~dB} / 30$ $\mathrm{mW}$, which is superior to that of the transmissive case $(\sim 34$ $\mathrm{dB} / 50 \mathrm{~mW}$ ) in [8] while using the same erbium-doped fiber The gain and NF for one-branch case further upgrade $\sim 2 \mathrm{~dB}$ and $\sim 3.5 \mathrm{~dB}$, respectively. From above, we may reasonably predict that the system net gain for the input signal power of about $-18 \mathrm{dBm}$ is $\sim 10 \mathrm{~dB}$ at $1532 \mathrm{~nm}$ and $\sim 8 \mathrm{~dB}$ at $1550 \mathrm{~nm}$ for eight-branch case; NF is $\sim 15 \mathrm{~dB}$ and $\sim 13 \mathrm{~dB}$ at $1532 \mathrm{~nm}$ and $1550 \mathrm{~nm}$, respectively. Considering the loss-less condition (i.e., system net gain $=0 \mathrm{~dB}$ ) for input signal of $-18 \mathrm{dBm}$

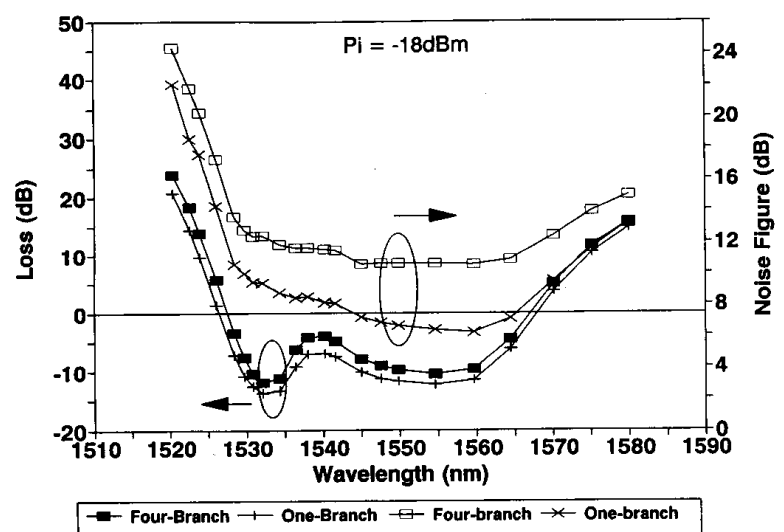

Fig. 3. The spectral loss and noise figure of this ARSC with signal input power of $-18 \mathrm{dBm}$ for one- and four-branch cases.

at $1550 \mathrm{~nm}$, we find the network size, $N$, can be obviously increased to about 128 by reason of the superfluous $8-\mathrm{dB}$ gain. In addition, though the network size $(N=64)$ is twice as that of the $32 \times 32$ transmissive amplified star coupler, the $8-\mathrm{dB}$ gain is still larger than that of the latter ( $\sim 3 \mathrm{~dB}$ gain) [8] for the same input signal power level of about $-20 \mathrm{dBm}$.

Fig. 4 shows the loss and NF characteristics of this ARSC, for different cross-channel signal powers per channel. We have an input signal of $1551.7 \mathrm{~nm}$ with small and large powers at channel \#1 and three cross-channel signals with wavelengths of $1549.7 \mathrm{~nm}, 1554 \mathrm{~nm}$, and $1557 \mathrm{~nm}$ at channel \#2, \#9, and \#17, respectively. If cross-channel signal powers get larger, the gain degrades more due to the gain compression effect, and, without doubt, the small cross-channel signal has little influence on the signal gain. For both small and large signal input powers, it is loss-less when cross-channel signal power per channel is less than $-10 \mathrm{dBm}$ and the net gains are $\sim 11.5$ $\mathrm{dB}$ and $\sim 3.7 \mathrm{~dB}$ for small and large input signal powers, respectively, in the small cross-channel signal region. As far as the NF is concerned, it is not influenced by cross-channel signals so easily as the gain is, and it is $\sim 10.3 \mathrm{~dB}$ and $\sim 12.5 \mathrm{~dB}$ for small and large signal, respectively, in small cross-channel signal power region.

The BER measurements plotted against, received signal power for three different cases and is shown in Fig. 5. With an input signal power of $-18 \mathrm{dBm}$ and no cross-channel signals at any input ports of the ARSC, the received power for $10^{-9}$ BER at $1.7 \mathrm{~Gb} / \mathrm{s}$ is $-34.5 \mathrm{dBm}$ at $1551 \mathrm{~nm}$. Thus, $\sim 1.3 \mathrm{~dB}$ power penalty arises with respect to the intrinsic case (i.e., without using the ARSC) owing to the generation of ASE noise in these four activated EDFA's. If compared with the commercial passive $64 \times 64$ star coupler, which has a loss of $\sim 20 \mathrm{~dB}$, we can get a power margin increment of about $21.7 \mathrm{~dB}$. The power penalty only increases to $\sim 2 \mathrm{~dB}$ with respect to the intrinsic case when ARSC is used with different cross-channel signals of about $-9 \mathrm{dBm}$ at channel \#2, \#9, and \#17 respectively; furthermore, an eye diagram of the detected signal for received optical power of $-32.5 \mathrm{dBm}$ is shown in the inset of Fig. 5. 


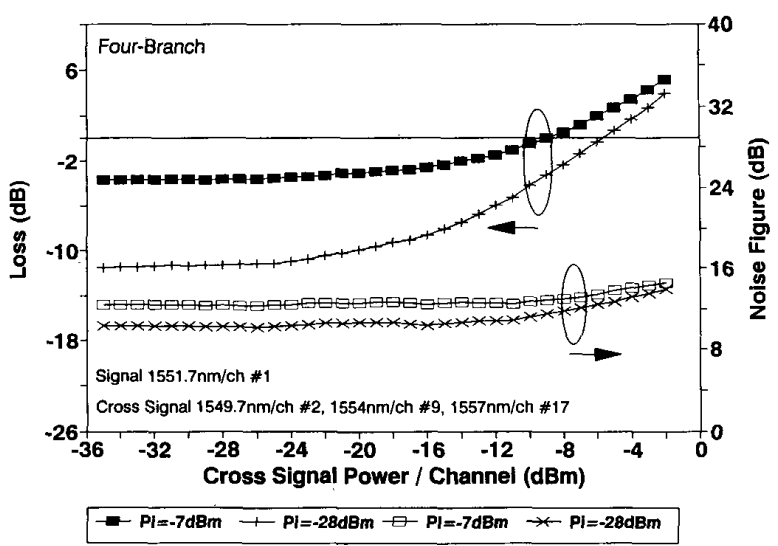

Fig. 4. The loss and noise figure of this ARSC for different input crosschannel signal powers.

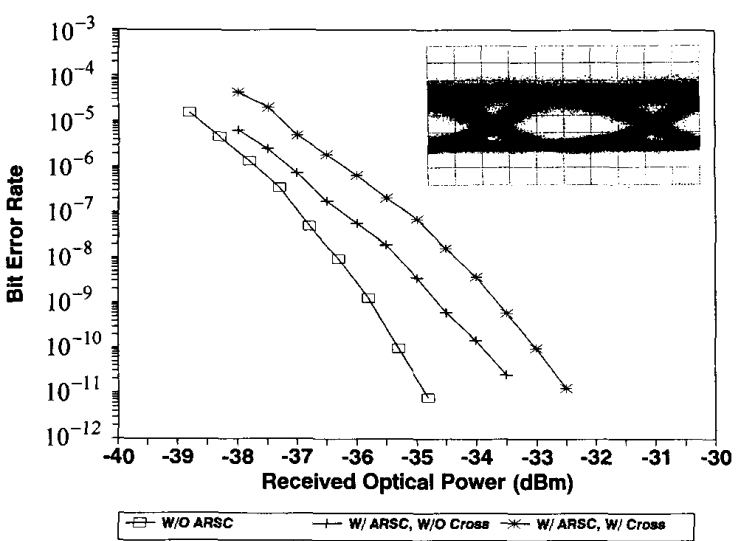

Fig. 5. BER measurements versus received optical power for (a) without ARSC, (b) with ARSC and without cross-channel signals, (c) with ARSC and with cross-channel signals. Inset: Eye diagram of (c) for received optical power of $-32.5 \mathrm{dBm}$.

Finally, the system saturated output signal power of this ARSC measured, is about $-3.8 \mathrm{dBm}$. This output signal power is approximately equal to the total saturated output power expected for multichannel applications. Further improvement of the saturation power may be achieved by using a more efficient EDF or increasing the pump power. The variations of the system insertion loss for the $32 I / O$ ports are just less than $3 \mathrm{~dB}$. The insertion loss variation mainly results from the characteristic differences among the four branches, including the $8 \times 1$ tree coupler, EDF length, pump power, etc. The uniformity property can be improved while carefully choosing optical components and optimizing each EDFA.

\section{CONCLUSION}

In conclusion, we have demonstrated a wide-band amplified reflective star coupler, by which the number of fibers and components required to implement a star network can be cost-effectively reduced to half. The loss of this star coupler can be fully compensated over a wide spectral range owing to the double-pass amplification and high pump power use efficiency. It also has high power margin increment of 21.7 $\mathrm{dB}$ with low power penalty of $1.3 \mathrm{~dB}$ and low cross-channel power penalty of $\sim 2 \mathrm{~dB}$. Compared with transmissive cases, the performance of loss compensation of amplified reflective star coupler is seen to be much better at the expense of a bit larger ASE noise. However, the power penalty resulting from the larger ASE noise is still low enough that multigigabit WDMA systems can accept it. Development of fiber amplifiers with high output saturation power will help realize large-size reflective star networks for WDMA network applications.

\section{ACKNOWLEDGMENT}

The authors would like to thank Dr. I. C. Jou, B. S. Jeng, Y. K. Tu, and J. W. Liaw for their encouragement throughout this work. The authors are also grateful to H. Y. Yang and his colleagues of MCVD group for supplying erbium-doped fibers.

\section{REFERENCES}

[1] C. A. Brackett, "Dense wavelength division multiplexing networks: Principle and application," IEEE J. Select. Areas Commun., vol. 8 no. 8 pp. $948-9641990$.

[2] Y. M. Lin, D. R. Spears, and M. Yin, "Fiber-based local access network architectures," IEEE Commun. Mag., vol. 28 no. 10, pp. 64-73, 1989.

[3] N. R. Dono et al., "A wavelength division multiple access network for computer communication," IEEE J. Select. Areas Commun, vol. 8 no. 6, pp. 983-994, 1990.

[4] A. A. M. Saleh and H. Kogelink, "Reflective single-mode fiber-optic passive star couplers," J. Lightwave Technol., vol. 6, no. 3, pp. 392-397, 1988.

[5] A. E. Wilner et al., "Star couplers with gain using multiple erbiumdoped fibers pumped with a single laser," IEEE Photon. Technol. Lett., vol. 3, no. 3, pp. 250-252, 1991 .

[6] Y. K. Chen and S. Chi, "WDM/FDM star coupler with gain using fiber amplifiers," Electron. Lett., vol. 29, no. 9, pp. 731-732, 1993.

[7] Y. K. Chen, S. Chi and J. W. Liaw, "Hybrid transmissive optical star couplers with gain using fiber amplifiers," IEEE Photonics Technol. Lett., vol. 5, no. 2, pp. 230-232, 1993.

[8] W. Y. Guo, Y. K. Chen, J. Y. Zeng and S. Chi, "Wideband $32 \times$ 32 star coupler module with gain," Electron. Lett., vol. 29 no. 20, pp. 1783-1784, 1993. 